\title{
HIV PREVENTION AND COMMUNITY ENGAGEMENT: 15 YEARS ON
}

\author{
Michael Salter \\ Community Liaison Officer \\ National Centre in HIV Social Research
}

The translation of research findings into evidence-based health promotion remains an important challenge in HIV prevention. Despite an increasing emphasis on establishing 'community engagement' and 'knowledge transfer' frameworks within health research, there has been little discussion of either the long-term nature of this work or the maintenance of the linkages between organisations and individuals that result from it. This article will provide an overview of the work of the National Centre in HIV Social Research (NCHSR) in this area, and will highlight the importance of reflexivity and adaptability in established knowledge transfer systems in the context of changing social, political and epidemiological environments.

\section{COMMUNITY ENGAGEMENT AT NCHSR}

From its very inception, NCHSR has been embedded in the network of community and government partnership that has characterised Australia's response to the HIV epidemic. NCHSR was established in 1990 following an approach to Macquarie University from members of the AIDS Council of NSW, who believed that social research had an important role to play in crafting effective HIV education and prevention. ${ }^{1}$ The initial project that gave rise to the Centre was the Social Aspects of the Prevention of AIDS (SAPA) study, a joint effort between researchers and community partners. ${ }^{2}$ The SAPA survey was developed collaboratively, and results were disseminated through community reports and feedback sessions.

NCHSR has a dual mandate to inform both the government and the community response to the threat of HIV, and community engagement is central to that task. Today, the framework of community engagement is similar to that established in the early 1990s, albeit in an expanded form. NCHSR is now host to two significant programs of community engagement and knowledge transfer. The commonwealth-funded Research Link project is now in its sixth year. Research Link has enabled NCHSR to hire a full-time Community Liaison Officer, who works with community educators and health promoters in translating social research into policy. NCHSR's hosting of the Consortium for Social and Policy Research on HIV, Hepatitis C and Related Diseases, funded by the NSW Department of Health, has also made it possible to develop a program of capacity-building workshops for workers in the HIV, hepatitis C and sexual health sectors, and a Masters program in health, sexuality and culture.

Research dissemination and knowledge transfer at NCHSR has traditionally occurred in the context of community engagement. If data collection is the flow of information to NCHSR through community partnerships, then knowledge transfer is the movement of research findings from NCHSR back to community partners. Knowledge transfer at NCHSR has been one aspect of a complex system of interactions between researchers, research participants and community stakeholders. This can be described as the 'community liaison' model of research transfer ${ }^{3}$ and, although our dissemination efforts have always had an impact beyond community stakeholders, it is an activity that we have traditionally targeted at those stakeholders in particular.

A long-term program of community engagement has had a number of outcomes, both for NCHSR and for our stakeholders. Community engagement demands a specific style of work from researchers that has benefits for NCHSR beyond the processes of knowledge generation and transfer. It requires a reflexive and team-orientated approach that has a ripple effect throughout the entire organisation. In addition, the involvement of stakeholders in research development provides a series of checks and balances that entrenches accountability and ultimately produces higher quality research by enabling new personal and professional experiences and insights.

Community engagement provides stakeholders with access to relevant and timely information, but perhaps a more important outcome is the research literacy that comes with proximity to the whole of the research process. Stakeholders are not the passive recipients of knowledge transfer, but partners in developing research ideas, writing grants, guiding projects and formulating conclusions. In any given project, community members and stakeholders provide not only the raw data, but also the expertise to understand and interpret that data. Through partnership with us and other research centres, the HIV community sector has developed a significant level of familiarity and comfort with research as a whole, and the demonstrated capacity to access research findings and incorporate them in practice.

\section{CHANGES IN THE WORK OF COMMUNITY ENGAGEMENT}

The increased currency of the 'evidence-based' paradigm has repositioned the knowledge transfer mandate within NCHSR, and challenged us to expand our dissemination efforts beyond the work of community engagement. Health providers are being encouraged to turn to research to both inform and justify their service delivery decisions ${ }^{4}$, and researchers are increasingly expected to engage policy makers and research consumers in both the construction and dissemination of research. ${ }^{5}$ This shift requires us to reach out to sexual health services, alcohol and other drug services, general practitioners, government employees, welfare workers and health care providers. The challenge is to integrate a broader 'knowledge transfer' agenda alongside 
our community engagement work, acknowledging that they are related but distinct endeavours. ${ }^{3}$

A number of important changes within our organisation, and in the context within which we work, also requires us to re-evaluate our community engagement and knowledge transfer strategies. Over the past five years, our research program has expanded beyond HIV and sexual health to include viral hepatitis and injecting drug use, both nationally and internationally. These new research foci have brought NCHSR into contact with a broader range of communities and populations across the country and the Asia-Pacific region. As the NCHSR research agenda expands, the work of community engagement takes on complex new dimensions. After a 16-year partnership, HIV community stakeholders demonstrate high levels of research literacy and significant buy-in to the NCHSR research program. In contrast, new stakeholders may be less familiar with social research, or may be working in contexts in which they have considerably fewer resources and less capacity to engage with research agendas, which has a direct influence on their receptivity to research. ${ }^{6}$ The early days of research partnerships are often characterised by negotiation over the ownership of both data and outcomes, and these are sensitive discussions that have consequences for both the relevance of research findings to affected communities and stakeholders, and the subsequent impact of these research findings.

Medical and epidemiological developments have also had an impact on both the direction of our research and the research needs of our partners. Since the introduction of highly active antiretroviral therapy in 1996, the HIV sector as a whole has had to adjust to the needs of an HIVpositive population who are now living with HIV rather than dying from AIDS-related conditions. In the view of some community partners, this has resulted in a perceived increased need for more research relating to health determinants and health systems implementation. Other stakeholders require information on health promotion, education, and the evaluation of intervention strategies. Others have interests in broader issues of gay and lesbian health. Some of these issues may intersect with the remit of the NCHSR research agenda; others may not. However, even the partial decoupling of our agenda from those of longstanding stakeholders requires delicate handling.

\section{RESPONDING TO CHANGE IN COMMUNITY ENGAGEMENT}

Identifying and responding to changes in community engagement has required an integrated planning response. Strategic planning is central to a well-coordinated community engagement strategy ${ }^{7}$, and in 2004 the Centre initiated a macro-level strategic review to provide an opportunity for institutional reflection on the specificity and clarity of our mission. The strategic plan, released in mid-2005, identified a number of key responses to changes in community engagement and knowledge transfer:

- Regular consultation frameworks: A collaborative research agenda, in and of itself, is a powerful strategy in promoting knowledge transfer and the application of research findings by research partners. ${ }^{8}$ The strategic planning process found a need for a more structured and planned approach to community consultation and NCHSR is in the process of establishing a schedule of regular, formal consultations.

- Memorandum of understanding: As part of the consultation process, NCHSR is drawing up a Charter for Community Engagement, a collective 'memorandum of understanding' that articulates the NCHSR approach to community engagement. As notions of 'community engagement' and 'knowledge transfer' shift and change, the purpose of the Charter is to articulate some formal commitments to community engagement as part of the research and dissemination process to ensure that NCHSR remains accountable to our community partners in these regards.

- Diversification of research output: Community reports have typically been targeted at education and health promotion managers, but mechanisms for disseminating research to workers at the coalface of HIV prevention have been limited. NCHSR is exploring the possibility of crafting shorter fact sheets that summarise relevant research findings in a manner accessible to both the HIV community sector and health workers more broadly. We are also discussing with community and health sector partners effective means of distributing these resources.

- Online dissemination: One of the central challenges in the dissemination of health research findings is simply getting information onto the desks of practitioners. ${ }^{6}$ The increasing prevalence of broadband enables online dissemination in a way that has not previously been possible. It enables us, for instance, to distribute fact sheets directly to workers, rather than rely on organisations to circulate printed reports on our behalf. However, online and offline research dissemination strategies need to be coordinated and complementary, which requires a strategic and managed approach to knowledge dissemination.

- Benchmarking and evaluation: Measuring community engagement is a challenge, but it dovetails closely with changes in the education and research sectors. ${ }^{5}$ Ideally, the evaluation of engagement strategies should be a cumulative study of the links between activities, outcomes and the overall context of community engagement. It could take the form of a yearly audit, in which community stakeholders are asked a series of questions about their awareness of opportunities to engage with NCHSR and their satisfaction with those mechanisms. We are presently looking into community 
engagement benchmarks and evaluation criteria, at a macro and organisational level, in terms of the quality, quantity and timeliness of engagement programs and policies.

While research suggests that our traditional modes of community engagement and dissemination (community reports, feedback sessions and seminars) are our most effective tools in the array of knowledge transfer techniques $^{6}$, community engagement and knowledge transfer are dynamic and adaptive processes. Taking stock of existing processes and emerging opportunities ensures the stability of stakeholder relationships and augments the effectiveness of dissemination strategies. The quality of research output is heightened when stakeholders have a central place in the generation of knowledge and understanding. Knowledge transfer, in its simplest sense, is the feedback mechanism in an economy of knowledge powered by engagement and collaboration.

\section{REFERENCES}

1. Kippax S, Kinder P. Reflexive practice: The relationship between social research and health promotion in HIV prevention. Sex Education 2002; 2: 91-104.
2. Kippax S, Connell RW, DowsettGW, Crawford J. Sustaining safe sex: Gay communities respond to AIDS. London: Falmer Press, 1993.

3. Mitchell A, Wals J. The community model of research transfer. In Lin V, Gibson B, editors Evidence-based health policy: Problems and possibilities. Melbourne: Oxford University Press, 2003: 263-71.

4. Black N. Evidence based policy: Proceed with care. British Medical Journal 2001; 323: 275-9.

5. National Health and Medical Research Council. A model framework for consumer and community participation in health and medical research. Canberra: NHMRC, 2005. Available at www.nhmrc.gov.au/publications/synopses/ r22syn.htm.

6. Del Santo T, Goldberg S, Choice P. Exploratory research in public social service agencies: An assessment of dissemination and utilization, Journal of Sociology and Social Welfare 2002; 24(4): 59-81.

7. Barnett K, Hattis P. Toward a more strategic approach to community engagement: A commentary. Journal of Health and Human Services Administration 2002; 25: 204-14.

8. Cameron R, Brown KS, Best A.J. The dissemination of chronic disease prevention programs: Linking science and practice. Canadian Journal of Public Health 1996, 87(S2), S50-S53. 露

\section{IMPROVING SUN PROTECTION BEHAVIOUR THROUGH EVIDENCE-BASED CAMPAIGNS}

\author{
Sandra Jones, Lyn Rees, Keryn Johnson \\ Centre for Health Behaviour and \\ Communication Research \\ University of Wollongong
}

\section{Anita Tang}

The Cancer Council New South Wales

Australia has the highest incidence of skin cancer in the world. Skin cancer is the most common form of cancer in Australia, with incidence rates outnumbering all other forms of cancer by more than three to one. The Australian health system spends more money on the diagnosis and treatment of skin cancer than on any other cancer, an estimated $\$ 420 \mathrm{~m}$ each year. ${ }^{1}$ The majority of these skin cancers could be prevented if the public could be persuaded to adequately protect themselves from the sun.

Social marketing is well placed to guide the development of sun protection programs, as it is orientated to achieving voluntary behaviour change at a group or community level. It is a knowledge discipline grounded in behavioural and communications theory that has developed from commercial marketing, with an underlying difference of being driven by a motivation to change consumer behaviour for the social or individual 'good', rather than for company profit. ${ }^{2}$ An understanding of the theory and practice of social marketing provides a foundation for the development of communication campaigns aimed at changing the community's health-related behaviour. However, many organisations that conduct communication campaigns do not adequately consider these factors in the development of their campaigns.

Social marketing has been used in the areas of smoking cessation, healthy eating, drug use and physical activity promotion. It has been used to a lesser extent for primary prevention of skin cancer. ${ }^{3}$ As a result, there is no documented evidence on how best to utilise social marketing within sun protection campaigns. In a specific application to sun protection, this project will investigate the use of social marketing and advertising communications theory in practice, and systematically apply the theory and research in the development of a demonstration campaign by the Cancer Council New South Wales.

This paper reports on work-in-progress and presents the proposed methodology and the results achieved in the first nine months of a three-year project. 